\title{
Fabrication of nanofibrous scaffolds for tissue engineering applications
}

\author{
H. CHEN, R. TRUCKENMÜLLER, \\ C. VAN BLITTERSWIJK and L. MORONI, \\ University of Twente, The Netherlands
}

\section{DOI: $10.1533 / 9780857097231.1 .158$}

\begin{abstract}
Nanofibrous scaffolds which mimic the structural features of a natural extracellular matrix (ECM) can be appealing scaffold candidates for tissue engineering as they provide similar physical cues to the native environment of the targeted tissue to regenerate. This chapter discusses different strategies to fabricate nanofibrous scaffolds for tissue engineering. We first describe three major methods for nanofibrous scaffold fabrication: molecular self-assembly, phase separation, and electrospinning. Then, approaches for surface modification of nanofibrous scaffolds including blending and coating, plasma treatment, wet chemical methods, and surface graft polymerization are presented. Finally, applications of nanofibrous scaffolds in tissue engineering are introduced.
\end{abstract}

Key words: electrospinning, self-assembly, phase separation, nanofibrous scaffolds, tissue engineering

\subsection{Introduction}

Tissue engineering aims to develop biological replacements through the integration of life science and engineering principles. In general, tissue engineering can be subdivided into three major approaches (Subbiah et al., 2005): (1) injection or transplantation of isolated cells to a defect site or an injured tissue, (2) delivery of tissue-inducing biomolecules (e.g. growth factors, peptides, polysaccharides) to a targeted tissue, and (3) growth and differentiation of specific cell types in three-dimensional (3D) scaffolds. Among these approaches, scaffold-based tissue engineering has become the most popular, due to the potential of current scaffold fabrication technologies to incorporate chemical, physical, and biological stimuli at different scales that can direct cell activity.

The extracellular matrix (ECM) usually provides structural support to the cells in addition to performing various other important functions. Many extracellular proteins have a fibrous structure with a diameter at the 
nanometer or sub-micrometer scales. For example, collagen, the most abundant ECM protein in our body, has a fibrous structure with a fiber diameter that ranges from 50 to $500 \mathrm{~nm}$. For successful and functional engineering of tissues and organs in scaffold-based approaches, an artificial scaffold should mimic the structure and biological function of native ECM as much as possible both in terms of physical cues and chemical composition. Therefore, researchers have put much effort into developing nanofibrous scaffolds that mimic the fibrillar structure of native ECM. Currently, there are three basic techniques available for generating nanofibrous scaffolds: molecular self-assembly, phase separation, and electrospinning. These techniques allow nanofibrous scaffolds that mimic the ECM physical structure to be developed with a number of degradable and non-degradable synthetic polymers, but additional modifications are desirable to present biological cues, which exhibit a positive effect on cell adhesion, proliferation, and differentiation. Furthermore, recent studies have shown that submicron and nanoscale topographies can also modulate cell activity, thus acting as 'synthetic' biological cues (Watari et al., 2011). Surface modifications of nanofibrous scaffolds include blending and coating, plasma treatment, wet chemical methods, and surface graft polymerization.

In this chapter, we describe nanofibrous scaffolds for tissue engineering. Processing technologies for achieving structural features resembling the extracellular matrix and approaches to improve cell-scaffold interactions will be introduced before discussing nanofibrous scaffold applications for tissue engineering.

\subsection{Methods for nanofibrous scaffolds fabrication}

Currently, there are three approaches available for the fabrication of nanofibers: (i) molecular self-assembly, (ii) phase separation and (iii) electrospinning. Although very different from each other, each method possesses its advantages and disadvantages (Table 6.1).

\subsubsection{Molecular self-assembly}

Molecular self-assembly has emerged as a useful approach for manufacturing scaffolds for tissue engineering (Goldberg et al., 2007). Unlike electrospinning, self-assembly can be defined as a spontaneous process to form ordered and stable structures through a number of non-covalent interactions, such as hydrophilic, electrostatic, and van deer Waals interactions (Philp and Stoddart, 1996; Hartgerink et al., 2001). The structural features of self-assembled materials can be tuned by controlling the kinetics, molecular chemistry, and assembly environment (e.g. pH, solvent, light, salt addition, 


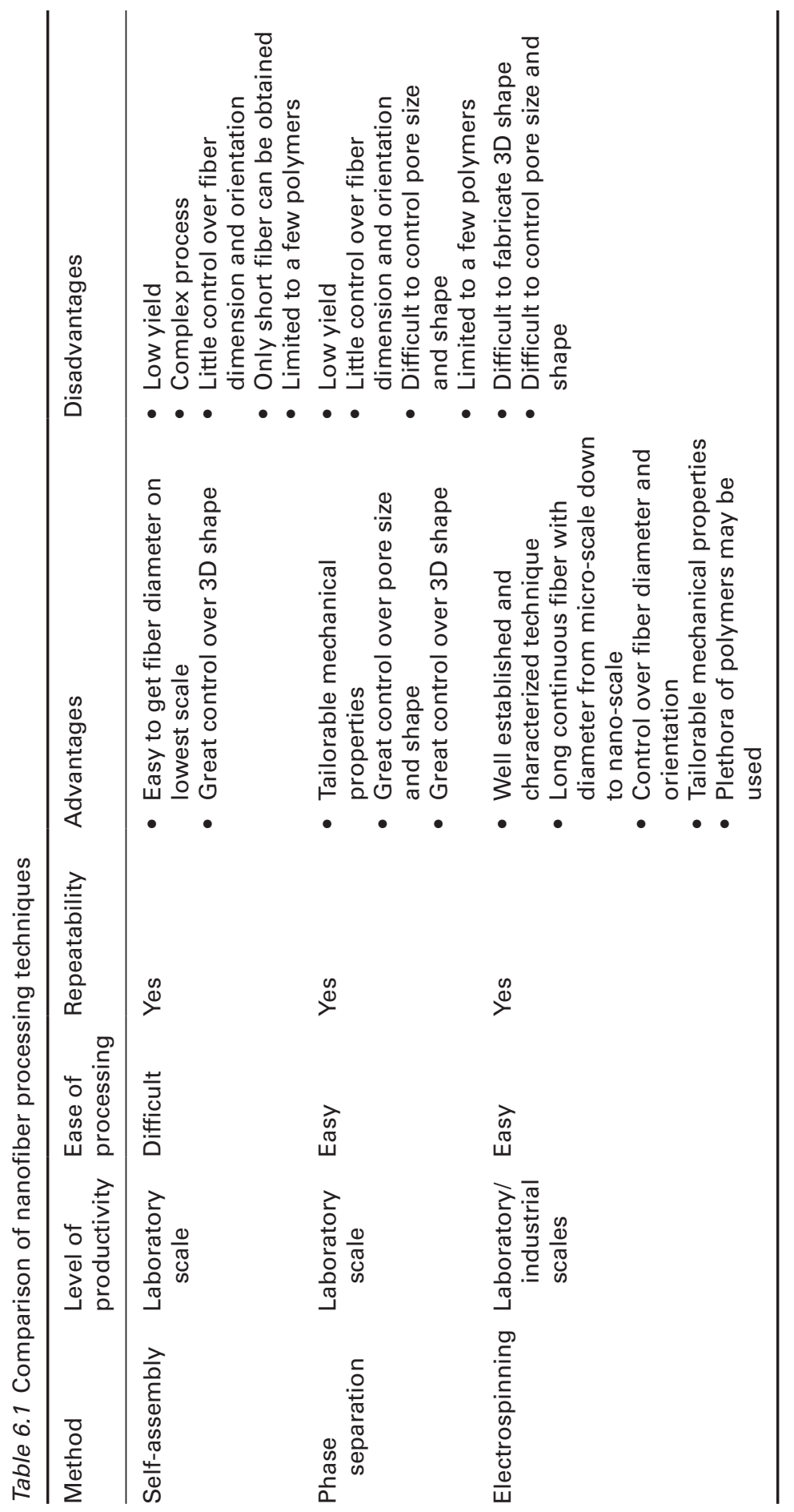


and temperature). The key challenge in self-assembly is to design molecular building blocks that can undergo spontaneous organization into a well-defined pattern that mimic the structural features of biological systems (Smith et al.,2008). Small building blocks, including small molecules, nucleic acids, and peptides, can self-assemble into nanofibrous structures. Among these building blocks, peptide-amphiphile (PA) units, which combine the functions of peptides with the characteristics of surfactants, have gained a lot of attention due to the versatility in their design for biological applications. The chemical structure of a representative PA molecule consists of four key structural features: a hydrophobic domain consisting of a long alkyl tail, a short peptide sequence capable of intermolecular hydrogen bonding, charged groups for enhanced solubility in water, and a bioactive epitope. Self-assembly of PA in water is governed by at least three major forces: hydrophobic interaction of alkyl tails, electrostatic repulsions between charged groups, and hydrogen bonding among peptide segments. The final structure of assembled PA reflects a balance of each force contribution (Cui et al., 2010a).

The structural characteristics of nanofibers and nanofibrillar systems resulting from molecular self-assembly can be tailored by controlling processing parameters. Bundles of aligned PA nanofibers were obtained, for example, by self-assembling PA within parallel channels (Hung and Stupp, 2007). By introducing hydrophilic amino acids in peptide segments, specific sequences of PA could self-assemble into nanobelts with fairly monodisperse widths in the order of $150 \mathrm{~nm}$ and lengths of up to $0.1 \mathrm{~mm}$; these can be used as cell culture systems and drug delivery carriers (Cui et al., 2009). Nanofibrous scaffolds were also fabricated from self-assembly of $\beta$-sheet peptides containing phenylalanine for controlled release (Cui et al., 2009; Zhao et al.,2010). These studies proposed that the position of aromatic moieties is a significant determinant for supramolecular self-assembling conformations. Self-assembled chitin fibers with diameters of $3 \mathrm{~nm}$ and $10 \mathrm{~nm}$ could be also obtained from dissolving in hexafluoro-2-propanol (HFIP) and $\mathrm{LiCl} / \mathrm{N}, \mathrm{N}$-dimethylacetamide (DMAC), respectively (Zhong et al., 2010). These observations demonstrated that nanofiber assembly occurs at a critical concentration and fiber morphology can be controlled by solution concentration and solution evaporation rate.

Molecular self-assembly is a fairly new technique for developing nanofibrous scaffolds which have been studied for a variety of tissue engineering applications including nerve (Guo et al., 2007), bone (Sargeant et al., 2008), and cartilage (Shah et al., 2010) regeneration. However, several technical hurdles still need to be addressed: first, it has not been demonstrated how to control the pore size and pore structure, which are important to allow for cell proliferation and migration. Secondly, their degradation profiles have not been systematically addressed (Smith et al., 2009). Further, most 
of the self-assembled scaffolds are mechanically weak and do not effectively sustain and transfer mechanical loadings to the cells and surrounding tissues.

\subsubsection{Thermally induced phase separation}

Another interesting method used for manufacturing nanofibrous scaffold is phase separation, which is a thermodynamic process involving the separation of phases due to physical incompatibility. Specifically, a homogeneous polymer solution becomes thermodynamically unstable under certain temperature conditions, and will form a polymer-rich phase and a polymer-poor phase. When the solvent is removed, the polymer-rich phase will solidify to a 3D structure while the polymer-poor phase will become the void space. The process of developing nanofibrous scaffold from phase separation typically consists of five major steps: (i) raw material dissolution, (ii) gelation, (iii) solvent extraction, (iv) freezing, and (v) drying (Ramakrishna, 2005). The pore morphology of nanofibrous scaffolds can be tuned by varying processing parameters including polymer concentration, phase separation temperature, and solvent/non-solvent exchange (Zhang et al., 2012). Furthermore, thermally induced phase separation can be combined with other processing techniques (e.g. particulate leaching and solid free-form fabrication) to generate scaffolds with complex porous structures and well-defined pore morphology (Holzwarth and Ma, 2011). For example, sodium chloride particles with diameters of 200-450 $\mu \mathrm{m}$ were mixed with a warm poly(lactic-co-glycolic acid)/tetrahydrofuran (PLGA/ THF) solution and then cooled to a preset gelation temperature. The composite gels formed were extracted with cold ethanol and washed with distilled water to remove the solvent and leach the salt particles. The samples were freeze-dried, resulting in a nanofibrous scaffold with a macroporous structure left behind from the leached salt (Mao et al., 2012). Nanofibrous poly(L-lactic acid) (PLLA) scaffolds have been developed by using phase separation to improve cell seeding, distribution, and mass transport (Woo et al., 2003). When compared with solid pore-walled PLLA scaffolds, nanofibrous scaffolds allowed an approximately 2 -fold increase in adhesion of osteoblast cells.

Thermally induced phase separation is a promising technique for developing nanofibrous scaffold with well-defined pore shape and pore size. Although this technique can be combined with other fabrication methods to control the final 3D structure, the drawbacks of this technique, including little control over fiber orientation and diameter, long fabrication time, and lack of mechanical properties, need to be addressed to further achieve a fine control of the resulting scaffolds at the macro-, micro-, and nano-scales. 
(a)

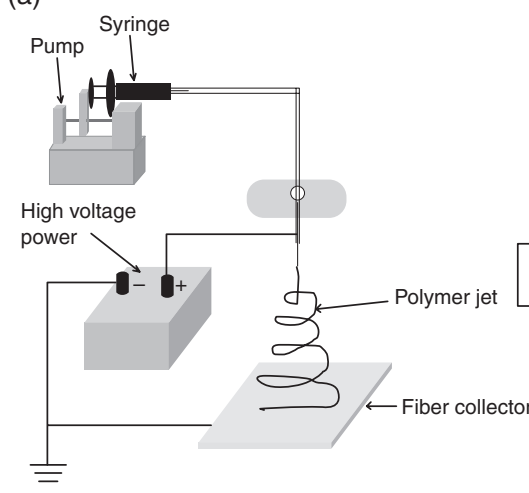

(b)

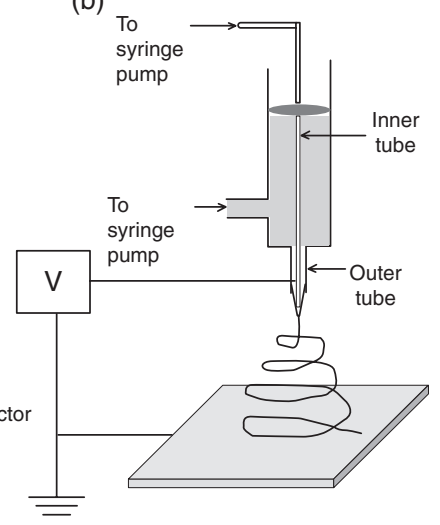

6.1 (a) Typical electrospinning set-up using a grounded static collector. (b) Configuration of the coaxial electrospinning set-up used for preparing core-shell structured nanofibers.

\subsubsection{Electrospinning}

Electrospinning is a versatile and well-established process capable of producing fibers with diameters down to the submicron or nanometer range (Prabaharan et al., 2011). A basic electrospinning setup (Fig. 6.1a) includes a high voltage electric source with positive or negative polarity; a syringe pump with capillaries or tubes to carry the solution (or melt) from a syringe to the spinneret; and a conducting collector (Sill and Von Recum, 2008). Under the electric field, the pulling force overcomes the surface tension of the polymer solution (or melt) and creates a charged jet that travels through the atmosphere allowing the solvent to evaporate, thus leading to the deposition of solid polymer fibers on the collector (Deitzel et al., 2002; Sill and Von Recum, 2008). The fiber formation and structure is affected by three general types of variable: solution properties (concentration, viscosity, conductivity, and surface tension), process factors (applied potential, collection distance, emitting electrode polarity, and feed rate), and environmental parameters (temperature, relative humidity, and velocity of the surrounding air in the spinning chamber) (Lee et al., 2003; Sill and Von Recum, 2008).

For many applications, it is necessary to fabricate a scaffold made of aligned nanofibers, as the anisotropy in topography and structure can have a great impact on mechanical properties and cell behavior. Currently, a number of collecting devices have been developed to attain aligned electrospun fibers. These collecting devices can be divided into three main categories according to the type of forces involved (Liu et al., 2011): (1) mechanical forces by using a rotating mandrel, (2) electrostatic forces by using parallel electrodes, and (3) magnetic forces by using parallel permanent magnets. 
Furthermore, electrospinning can be used for fabricating scaffold with complex architectures such as stacked arrays and tubular conduits. Stacked arrays of nanofiber scaffolds can be achieved by multilayering electrospinning. Aligned nanofibers were stacked into multilayered films with a controllable hierarchical porous structure by depositing fibers on an insulating substrate (e.g. quartz) onto which an array of electrodes have been patterned (Li et al., 2004). Tubular conduits made of electrospun fibers can be fabricated by depositing fibers over a small diameter rod as a collector. These electrospun conduits are usually applied in vascular or neural tissue engineering since they mimic the structure of these tissues. For instance, electrospun fiber conduits were manufactured with a length of $12 \mathrm{~cm}$ and a thickness of $1 \mathrm{~mm}$ via electrospinning a mixture of collagen type I, elastin, and poly(D,L lactide-co-glycolide) on a rotating rod of diameter $4.75 \mathrm{~mm}$ (Stitzel et al., 2006). Compliance tests demonstrated that the electrospun fiber conduit possesses a diameter change of $12 \sim 14 \%$ within the physiologic pressure range, a compliance behavior similar to that of a native vessel with a diameter change of $9 \%$. Burst pressure testing results showed that the burst pressure for the electrospun fiber conduit was $1425 \mathrm{mmHg}$ or nearly 12 times that of normal systolic pressure.

In order to tailor the structures of resultant fibers, the modification of electrospinning set-ups have been carried out not only on the fiber collector, but also on the spinneret. Coaxial spinnerets have been designed by many researchers for various aims in electrospinning (Fig. 6.1b). Using this technology, core-shell nanofibers can be achieved. By using a spinneret with double coaxial capillaries, two components can be fed through different coaxial capillary channels to generate composite nanofibers in the form of a core-sheath structure (He et al., 2006; Zhang et al., 2006; Venugopal et al.,2008). Some difficult-to-process polymer solutions can be also electrospun to form an ultrafine core within the shell of spinnable polymers. Thus, when the polymer sheath is removed, the desired polymer nanofiber core is retained (Wang et al., 2006). Using a similar concept, the core solution can be removed instead of the polymer sheath, resulting in hollow nanofibers. For example, an ethanol solution containing poly(vinyl pyrrolidone) (PVP) and tetraisopropyl titanate $\left(\mathrm{Ti}(\mathrm{OiPr})_{4}\right)$ was used as sheath solution while mineral oil was used as core solution. After simultaneous ejection through the inner and outer capillaries, hollow nanofibers were formed through removal of the inner core mineral oil phase (Li and Xia, 2004).

Shell-core and hollow nanofibers have shown their potential in tissue engineering and drug delivery due to their unique architecture and properties. Biologically active agents can be encapsulated inside a polymer shell to form a reservoir-type drug delivery device. Therefore, the polymer shell would offer a temporal protection for the bioactive agents and control their sustained release (He et al.,2006). Tissue regeneration processes can be also 
facilitated via introduction of biomolecules (e.g. growth factors) into the core of shell-core nanofibrous scaffolds and, eventually, the release of multiple factors can be envisioned by adding different compounds in the shell and in the core fibers. Electrospun fibers with high surface to volume ratio and structures resembling ECM have shown great potential for applications in tissue engineering and drug delivery (Cui et al., 2010b). Yet, a fine control over nanofiber population distribution is still lacking and is so far a limit in obtaining standardized scaffolds.

\subsection{Surface modification of nanofibrous scaffolds}

Surfaces play a vital role in biology and medicine, as most biological reactions take place at the interface between biological systems (Castner and Ratner, 2002). In tissue engineering, the chemical and physical characteristics of the biomaterial surface strongly impact on cell behavior, such as migration, attachment, and proliferation (Jiao and Cui, 2007). Although various degradable and non-degradable synthetic polymers have been used as tissue engineering scaffolding materials, a shortcoming of these materials is their lack of biological recognition (Smith et al., 2008). Currently, several techniques have been developed to modify the scaffold surface including physical coating and blending, plasma treatment, graft polymerization, and wet chemical methods (Fig. 6.2).

\subsubsection{Physical coating or blending}

Perhaps the most straightforward and convenient means of modifying a polymer surface is blending functional molecules and active agents into the bulk polymer or just coating it on the polymer surface. Two or more materials are physically blended together to attain a new biocomposite with superior surface and/or mechanical properties. PLLA nanofibers with hydroxyapatite (HA) particles exposed on their surface were achieved by electrospinning different blended solutions (Sui et al., 2007; Luong et al., 2008). These composite fibers not only promoted osteoblast adhesion and growth, but also exhibited superior tensile properties as compared to the pure PLLA fibers, due to the internal ionic bonding between calcium ions in HA particles and ester groups in PLLA (Deng et al., 2007). Jun et al. (2009) developed electrically conductive blends of poly(L-lactide-co-caprolactone) (PLCL) and polyaniline (PANi) submicron fibers and investigated their influence on the induction of myoblasts into myotubes. Incorporation of PANi into PLCL fibers significantly increased the conductivity. In addition, the tensile strength and elongation at break of PLCL fibers increased as the concentration of PANi in the fibers decreased, while the Young's modulus exhibited an 
(a)

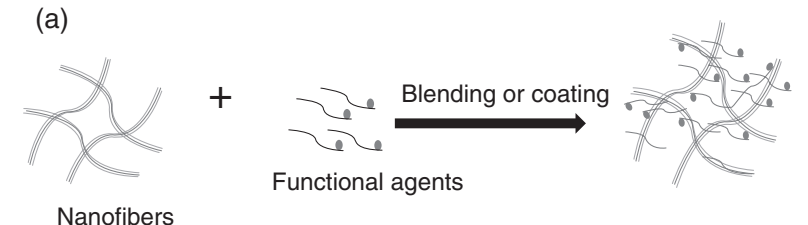

(b)
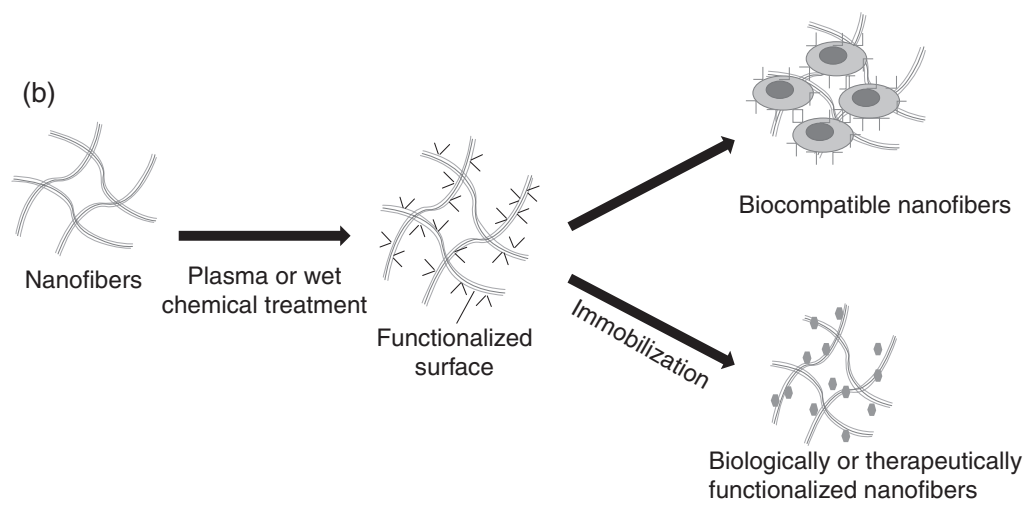

(c)

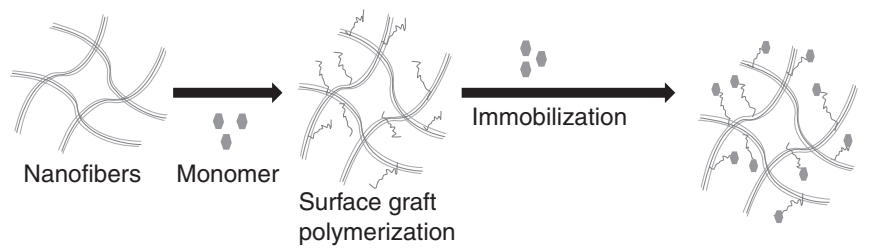

6.2 Surface modification techniques of nanofibrous scaffolds.

(a) Blending and coating; (b) plasma or wet chemical treatment;

(c) surface graft polymerization.

opposite trend. Taken together, these examples show that blending modifies not only the surface properties of substrate but also their bulk properties.

Coating is another physical approach of functionalizing nanofibrous scaffolds. Although this technique is limited in terms of controllability and maybe coatings prone to detachment from the scaffold, it remains one of the easiest and most convenient ways to functionalize the biomaterial surface. In general, a material containing the desired functional properties is used to coat nanofibrous scaffolds, aiming at improving their surface biocompatibility and enhancing cell-scaffold interactions. In recent years, coating of functional molecules onto polymers was significantly developed by combining new techniques including coaxial electrospinning and layer-by-layer assembly (LbL). Collagen-coated poly( $\varepsilon$-caprolactone) (PCL) nanofibers were developed by a coaxial electrospinning technique with collagen and PCL as inner and outer solutions, respectively, and cultured with human 
dermal fibroblasts (HDF) (Zhang et al., 2005). The results demonstrated that collagen-coated PCL nanofibers from coaxial electrospinning showed higher efficiency in favoring cell proliferation compared to collagen-coated PCL nanofibers prepared by soaking the PCL matrix in a $10 \mathrm{mg} / \mathrm{mL}$ collagen solution. The mechanism behind LbL coatings is that positively and negatively charged macromolecules are alternatively introduced onto the substrate surface via strong electrostatic interaction (Ramakrishna, 2005). An environmentally benign surface modification process for poly(ethylene terephthalate) (PET) films was demonstrated by fabricating composite coatings through LbL assembly with cellulose and chitin nanofibrils (Qi et al., 2012). The modified PET films exhibit high light transparency, flexibility, surface-hydrophilicity, and specific nanoporous structures.

\subsubsection{Plasma-based surface modification}

Plasma, also named the fourth state of matter, is a gaseous mixture of particles containing charged particles, excited neutral atoms and molecules, radicals, and UV photons (Denes and Manolache, 2004). Generally, plasma can be subdivided into two categories according to the temperature of particles: thermal plasma and non-thermal plasma. Non-thermal plasma, which is composed of low temperature particles has been commonly employed to modify the surface of heat-sensitive materials such as biodegradable polymers (Morent et al., 2011). By using this technique, diverse functional groups can be effectively incorporated on the target surface of biodegradable polyesters to improve surface adhesion and wetting properties. For example, typical plasma treatment with different gaseous atmospheres such as oxygen, air, and ammonia can introduce carboxyl groups or amine groups to the target surface (Zhu et al., 2005).

After introduction of specific functional groups on the surface of substrates by plasma treatment, synthetic and natural macromolecules could further immobilize monomers (e.g. extracellular matrix protein components) on their surface to enhance cellular adhesion and proliferation. This process is called plasma grafting. Nanofibrous scaffolds composed of PLLA and PLGA were fabricated by electrospinning (Park et al., 2007b). Their surfaces were treated with oxygen plasma treatment and grafted with hydrophilic acrylic acid. Such surface-modified scaffolds were shown to improve fibroblast attachment and proliferation in vitro. Plasma polymerization, another plasma-based surface modification approach, is distinct from plasma grafting by coating the substrate instead of covalent binding species to a plasma-treated polymer surface (Barry et al.,2006; Morent et al., 2011). Under plasma conditions, gaseous or liquid monomers are converted into reactive fragments which can, in turn, recombine to form a polymer film 
which is deposited onto the substrate exposed to the plasma. To gain more insights into plasma polymerization processes, various $-\mathrm{C}: \mathrm{H}: \mathrm{N}$ thin films were deposited on glass under different $\mathrm{NH}_{3} / \mathrm{C}_{2} \mathrm{H}_{4}$ gas ratios, power inputs (W), and gas flow rates (F) (Guimond et al.,2011). The results demonstrated that the film growth is determined by the ratio between the reaction parameters power inputs $\mathrm{W}$ and gas flow rates $\mathrm{F}$, and the energy dissipated at its surface during the deposition given by ion flux times mean ion energy per deposition rate.

Plasma treatment, an effective and solvent-free technique, is commonly used for surface modification. The disadvantage of plasma treatment is that only localized surface areas can be treated (in depth from several hundred angstroms to $10 \mathrm{~nm}$ ) without changing the bulk properties of the polymers (Jiao and Cui, 2007). Currently, most plasma-based surface modification is performed on two-dimensional (2D) polymer substrates, and in some cases on $3 \mathrm{D}$ porous scaffold.

\subsubsection{Wet chemical methods}

The cellular response to a biomaterial may be enhanced in synthetic polymer formulations by mimicking the surface roughness created by the associated nano-structured ECM components of natural tissue. PLGA films with nano-structured surface features were developed by treating them in a concentrated $\mathrm{NaOH}$ solution (Miller et al., 2004). The results from cell experiments indicated that $\mathrm{NaOH}$-treated PLGA enhanced vascular smooth muscle cell adhesion and proliferation compared to conventional PLGA. This surface modification method is used not only on 2D film surfaces but also on 3D constructs. Chen et al. (2007) obtained PCL membranes with nanofibrous topology by coating the membrane surface with electrospun nanofibers. When the nanofiber-modified PCL was treated with $5 \mathrm{M} \mathrm{NaOH}$ for 3h, a favorable 3T3 fibroblast cells morphology and strong cell attachment were observed on the modified surface, possibly due to the unique hydrophilic surface topography. Electrospun PLLA nanofibrous scaffolds were used as a matrix for mineralization of hydroxyapatite (Zhu et al., 2002). Carboxylic acid groups were introduced on the surface of PLLA scaffolds by hydrolysis in $\mathrm{NaOH}$ aqueous solution. Since calcium ions can bind to the carboxylate groups on the fiber surface, a significant improvement of the mineralization process was observed on modified PLLA electrospun scaffolds.

Although wet chemical methods have been widely applied to modify the surface wettability of biomaterials or to generate new functionalities, some major drawbacks should be noted. This modification technique can cause a partial degradation and scissions of the polymeric chains, resulting in loss 
of mechanical properties and a faster degradation process (Morent et al., 2011). In addition, the use of hazardous organic solvents might affect cell viability.

\subsubsection{Surface graft polymerization}

Among the surface modification techniques, surface graft polymerization has emerged as a simple, effective, and versatile approach in the surface functionalization of polymers for a wide variety of applications (Ramakrishna, 2005). Grafting exhibits several advantages (Ramakrishna, 2005; Pettikiriarachchi et al., 2012). Firstly, there is the ability to modify the polymer surface to possess desired properties through the choice of different monomers. Secondly, the ease and controllable introduction of graft chains with a high density and exact location of graft chains to the surfaces is possible without changing the bulk properties. Finally, a stable chemical bond is formed between the nanofiber surface and the preformed polymer, which offers endurance of the functional component, in contrast to physically coated polymer chains.

Surface graft polymerization is often initiated with plasma discharge, ultraviolet (UV) light, gamma rays, and electron beams (Yoo et al., 2009; Pettikiriarachchi et al., 2012). Chua et al. (2005) investigated how a functional poly( $\varepsilon$-caprolactone-co-ethyl ethylene phosphate) (PCLEEP) nanofibrous scaffold with surface-galactose ligand influenced behavior of rat hepatocytes. The modification of nanofibrous scaffolds was achieved by conjugating galactose ligands to a poly(acrylic acid) spacer UV-grafted onto the fiber surface. The functionalized nanofibrous scaffolds exhibited the unique property of promoting hepatocyte aggregates within the mesh and around the fibers. Furthermore, hepatocyte functions are maintained on these functional nanofiber substrates, similar to a galactosylated-film substrate configuration.

The grafting of extra cellular protein like collagen, arginine-glycine-aspartic acid (RGD) peptides, and gelatin on nanofiber surfaces has become a popular method to develop biomimicking-tissue scaffolds. For example, the modification of electrospun polyethylene terephthalate (PET) nanofibers involved fiber treatment with formaldehyde to generate hydroxyl groups on the surface, followed by graft polymerization of methacrylic acid monomers initiated by Cerium (IV). Gelatin was then immobilized on the nanofibers by conjugation to the carboxylic acid moieties on their surface (Ma et al., 2005). The grafting of gelatin enhanced the adhesion and spreading of cells on nanofibrous scaffolds. In another study, gelatin was grafted onto PLLA scaffolds via aminolysis of the PLLA fibers followed by glutaraldehyde coupling (Cui et al., 2008). 


\subsection{Applications of nanofibrous scaffolds in tissue engineering}

The characteristics of nanofibrous scaffolds that render them promising candidates for tissue engineering include high surface area and porosity, as well as the similarity of their fibrous structure to the physical features of natural ECM (Ma and Zhang, 1999; Park et al., 2007a; Agarwal et al., 2009; Pettikiriarachchi et al., 2012). These features result in a more biologically compatible environment in which cells can grow and perform their regular functions. Therefore, nanofibrous scaffolds have been widely used as scaffolds for tissue engineering such as neural, cartilage, vascular, and bone tissue engineering (Table 6.2).

\subsubsection{Nanofibrous scaffolds for neural tissue engineering}

The nervous system coordinates the action of humans and transmits signals between different parts of the body. However, the nervous system has very limited capacity to repair itself after an injury. As a result, patients who have injures or traumas in the nervous system often suffer from the loss of sensory or motor function, and neuropathic pains (Venugopal and Ramakrishna, 2005; Prabaharan et al., 2011). In order to facilitate the regrowth of nerves, many therapeutic approaches have been attempted. One of the most promising approaches is to adopt a neural tissue engineering strategy that employs a scaffold or conduit to facilitate nerve regeneration.

Yang et al. (2005) studied aligned and random PLLA electrospun nanofibrous scaffolds for the purpose of neural tissue engineering. Their results indicated that neural stem cell (NSCs) elongated and their neurites outgrew along the direction of the fiber orientation of the aligned nanofibers (Fig. 6.3). Furthermore, NSCs on aligned nanofibrous scaffolds showed a higher rate of differentiation than on microfibers. Thus, the aligned PLLA nanofibrous scaffolds showed potential for application in neural tissue engineering.

Arginine-alanine-aspartate (RAD)16-I are the most commonly used peptides in self-assembled peptide scaffolds for neural cell culture (Semino et al., 2004; Silva et al., 2004; Gelain et al., 2006). Semino et al. (2004) developed RAD16-I self-assembled peptide scaffolds as a 3D culture system for cell encapsulation. Primary rat hippocampal cells were cultured on top of the self-assembled nanofibrous scaffold. Immunochemistry assays showed that glial cells and neurons increasingly migrated into the peptide scaffold to an approximate depth of 400-500 $\mu \mathrm{m}$ from the edge of the tissue in 1 week of cultures. Furthermore, mitotic activity of neural cells was maintained for 3 days after migration, which was attributed to the presence of the nanofibrous 


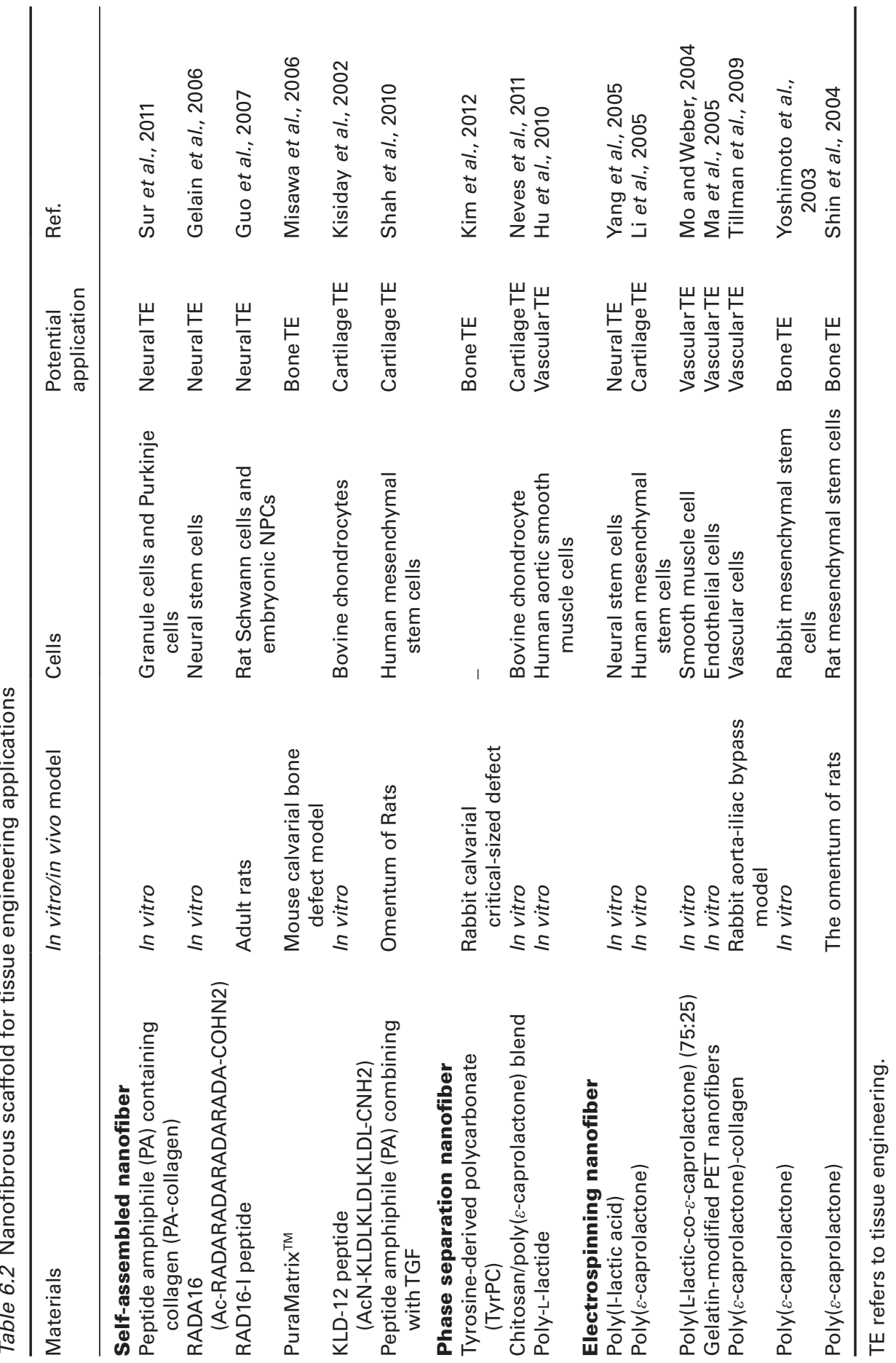



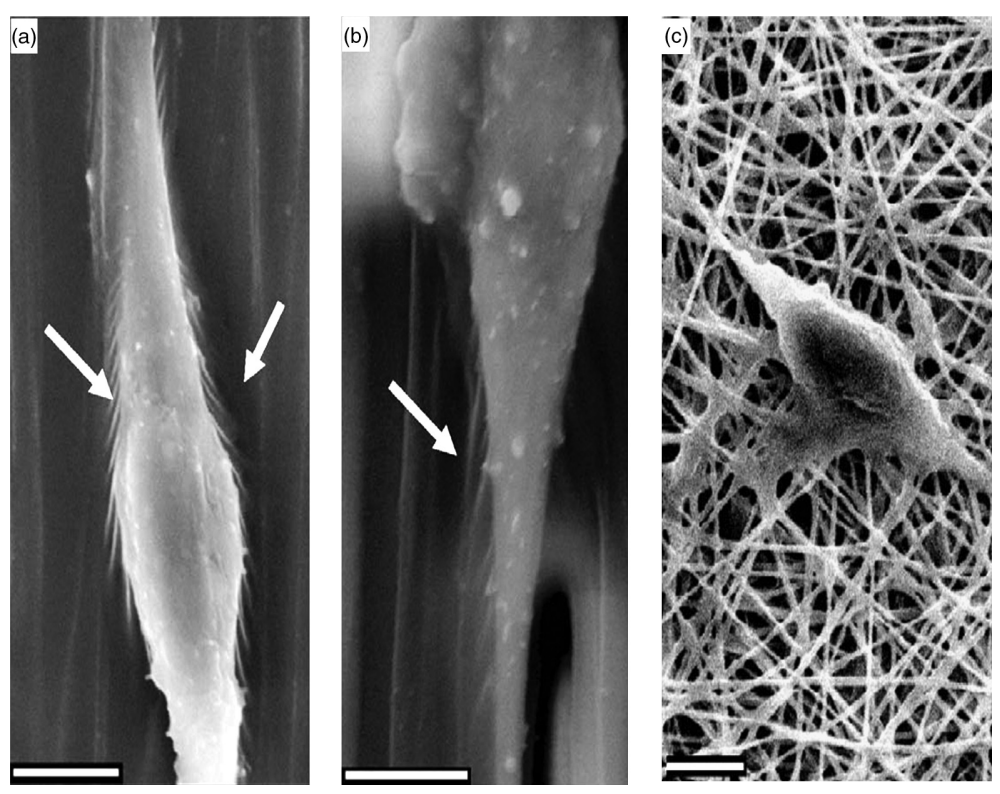

6.3 SEM micrographs of NSCs seeded on (a) nano-scale aligned fibers; (b) micro-scale aligned fibers; (c) nano-scale random fibers for 2 days showing the cell-matrix adhesion between the NSCs and the PLLA fibers. Arrows show some filament-like structures extend out from the NSC cell body and neurites and attach to the nanofibers. Bar $=5 \mathrm{~mm}$ (Yang et al. 2005).

scaffold environment resembling the native ECM. These results revealed that self-assembled peptide nanofibrous scaffolds are a potential substrate for supporting neural tissue regeneration. A hybrid nanofibrous matrix with homogeneous fiber diameter of 20-30 nm was designed by self-assembly of PA with the ability of presenting laminin epitopes (IKVAV or YIGSR) and collagen molecules (Sur et al., 2011). Granule cells and Purkinje cells, two major neuronal subtypes of the cerebellar cortex, were cultured on the hybrid scaffold. The result showed the ability to modulate neuron survival and maturation by easy manipulation of epitope densities.

In addition to in vitro experiments, self-assembled nanofibrous scaffolds have been transplanted into animal models for the treatment of central nervous system injuries. Self-assembled RAD16-I peptide scaffold in combination with adult rat Schwann cells and embryonic neural progenitor cells (NPCs) were transplanted into adult rats (Guo et al., 2007). The results indicated that the scaffolds integrated well with the host tissue with no obvious gap between the implants and the injured sites. A noteworthy observation from these experiments was that a large amount of host cells migrated into the scaffolds and extensive blood vessel formation was observed in the implants. 


\subsubsection{Nanofibrous scaffolds for cartilage tissue engineering}

Cartilage, a predominantly avascular, alymphatic, and aneural tissue, is composed of chondroblasts embedded within a dense extracellular matrix (Chung and Burdick, 2008). Cartilage is classified into three types: elastic cartilage, hyaline cartilage, and fibrocartilage. Cartilage damage resulting from developmental abnormalities, trauma, aging related degeneration, and joint injury cause disability and extensive pain (Venugopal and Ramakrishna, 2005; Wang et al., 2005). Due to its limited capacity to self-regenerate, cartilage is an ideal candidate for tissue engineering. In cartilage tissue engineering, chondrocytes and mesenchymal stem cells (MSCs) are commonly used for cartilage repair. Electrospun PCL nanofibrous scaffolds were seeded with human bone marrow-derived MSCs to investigate their ability to support in vitro MSC chondrogenesis (Li et al., 2005). The results demonstrated that PCL nanofibrous scaffolds in the presence of transforming growth factor- $\beta 1$ (TGF- $\beta 1$ ) induced a differentiation of MSCs to chondrocytes comparable to pellet cultures. Although no inductive property of PCL nanofibrous scaffolds was observed, these meshes have better mechanical properties than cell pellets making them suitable for cartilage tissue engineering. Kisiday et al. (2002) investigated a self-assembling peptide hydrogel scaffold for cartilage regeneration. They combined the peptide KDK-12 with bovine chondrocytes and allowed them to self-assemble into a hydrogel. Their results indicated that chondrocytes proliferated well and maintained a chondrocytic phenotype in the hydrogel. Cells were able to produce cartilage-like ECM which was rich in type-II collagen and proteoglycans. Peptide amphiphiles (PAs) synthesized with a peptide binding sequence to TGF- $\beta 1$ were designed to form nanofibers via self-assembly for cartilage regeneration (Shah et al., 2010). The study demonstrated that these scaffolds support the survival and promote the chondrogenic differentiation of human mesenchymal stem cells. In vivo experiments showed that these scaffolds promoted regeneration of articular cartilage in a rabbit model with, or even in the absence of, exogenous growth factor (Plate VII, see color section between pages 264 and 265).

\subsubsection{Nanofibrous scaffolds for vascular tissue engineering}

Blood vessels perform a very important function of carrying and transporting blood to and from heart. In addition, they are also important places for the exchange of water and other chemicals between blood and the tissue (Ramakrishna, 2005; Cui et al., 2010b). Mo and Weber (2004) developed an 
aligned electrospun nanofibrous scaffold from biodegradable poly(L-lactic acid-co-e-caprolactone) (PLLA-CL) (75:25) with the goal of developing constructs for vascular tissue engineering. The fabricated nanometric fibers resembled the dimensions of natural ECM, possessed mechanical properties comparable to human coronary artery, and supported smooth muscle cell adhesion and proliferation well.

A major reason for graft failure is that the graft surface is only partially covered by endothelial cells following a degenerative state. To overcome this problem, PET nanofibrous scaffold were developed by electrospinning and their surfaces were modified by grafting gelatin. Their study indicated that gelatin-modified PET nanofibers were favorable for spreading and proliferation of endothelial cells and maintained cell phenotype (Ma et al., 2005). Tillman et al. (2009) studied the in vivo stability of electrospun PCL-collagen scaffolds in vascular regeneration in a rabbit aortal-iliac bypass model. Their findings suggested that PCL-collagen scaffolds maintained enough mechanical strength for cell growth and other physiologic conditions.

\subsubsection{Nanofibrous scaffolds for bone tissue engineering}

Bone is a composite material made from an organic phase of collagen and glycoproteins, and an inorganic phase primarily consisting of hydroxyapatite (HA) (Rhoades and Pflanzer, 1996). The organic phase provides the resilient nature of bone and the inorganic minerals are responsible for bone hardness (Ramakrishna, 2005; Nisbet et al., 2009). Bone loss may be caused by trauma, fractures, periodontitis, cancer, infectious disease, and osteoporosis (Kimakhe et al., 1999). Presently, many approaches have been developed for bone regeneration, such as autografts, metal implants, and allografts. However, all of these methods have obvious drawbacks. For example, autografts present problems associated with a limited donor source as well as a secondary surgery site. Therefore, tissue engineering approaches are presently being investigated as a promising method for bone regeneration.

Yoshimoto et al. (2003) studied the potential of non-woven PCL scaffolds generated by electrospinning for bone tissue engineering. MSCs derived from bone marrow of neonatal rats were seeded on the nanofibers. The results demonstrated that MSCs migrated into the scaffolds and produced abundant ECM. Based on this study, Shin et al. (2004) implanted MSCs along with PCL nanofibrous scaffolds into the omentum of rats. Their study indicated ECM formation throughout the scaffolds along with the evidence of mineralization and type I collagen synthesis. When HA is incorporated into nanofibrous scaffolds it not only improves the mechanical properties, but also creates more biomimetic constructs. Either nanoparticles of HA, morphogenetic proteins (e.g. BMP-2) or both were incorporated into 

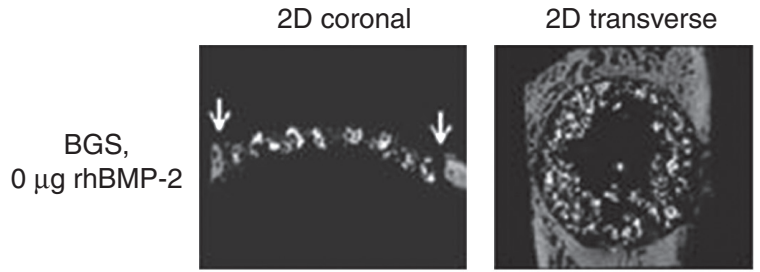

3D reconstruction
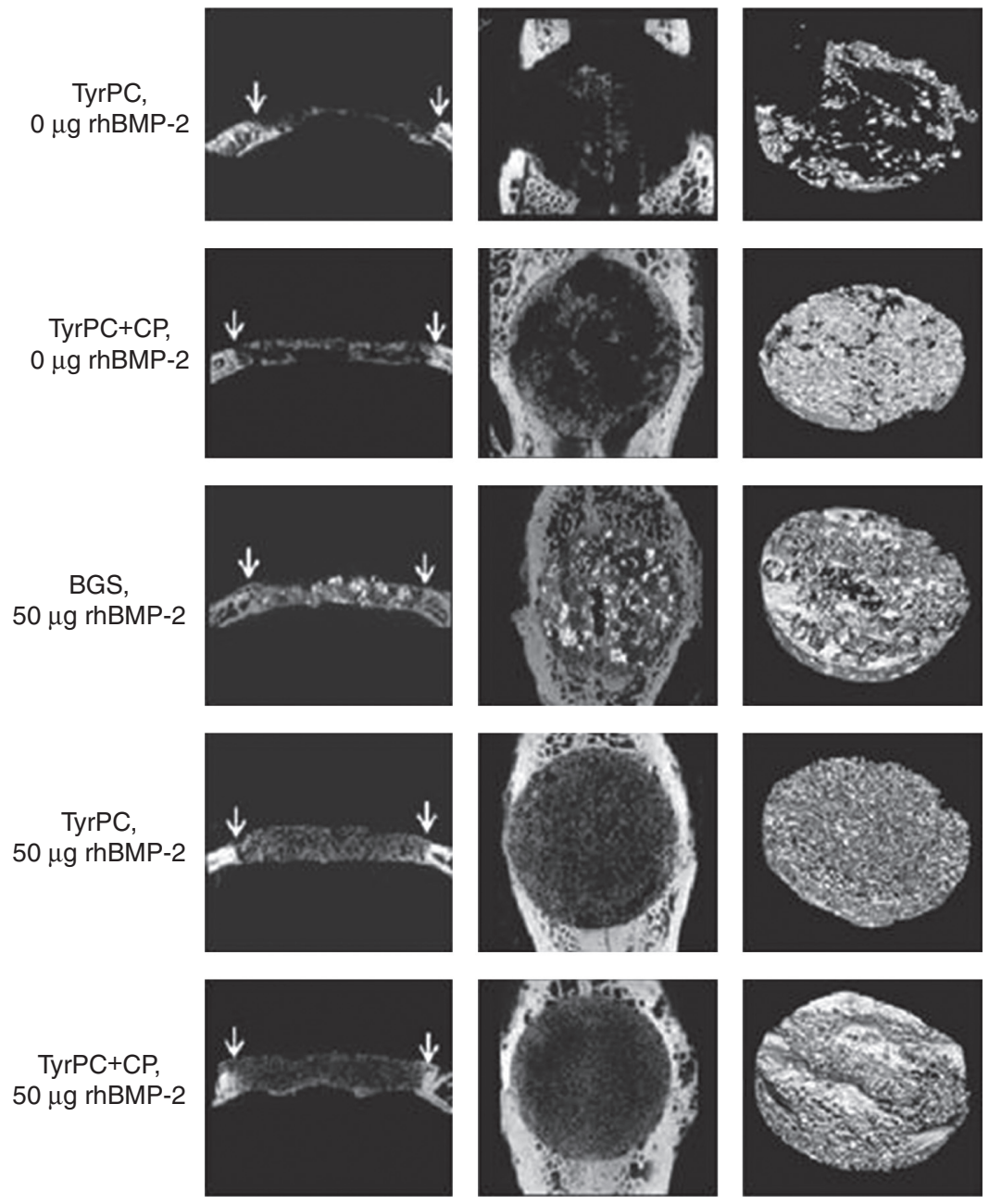

6.4 Representative microcomputed tomography images of bone regeneration in the rabbit model at 6 weeks post-implantation. White arrows in the first column identify the defect site. Remaining b-tricalcium phosphate fragments are identified in the 2D transverse images of the bone graft substitute (BGS) scaffolds (Kim et al., 2012). 
electrospun nanofibrous scaffolds of silk fibroin (Li et al., 2006). Scaffolds were seeded with human MSCs for 31 days in osteogenic medium. HA nanoparticles were associated with improved bone formation. Furthermore, when silk fibroin scaffolds were combined with both nanoparticles of HA and BMP-2, they were associated with the highest observed mineralization.

Porous three-dimensional tyrosine-derived polycarbonate (TyrPC) scaffolds were developed for bone regeneration using a combination of salt-leaching and phase separation techniques (Kim et al., 2012). The TyrPC scaffolds treated with or without recombinant human bone morphogenetic protein-2 (rhBMP-2) were implanted in a rabbit calvarial critical-sized defect model for 6 weeks. The in vivo studies showed that TyrPC scaffolds treated with rhBMP-2 or coated with calcium phosphate alone promoted bone regeneration at 6 weeks post-implantation (Fig. 6.4). Moreover, TyrPC polymeric scaffold, even without addition of any biological agents, induced similar bone regeneration to a commercially available bone graft substitute.

\subsection{Conclusion}

Mimicking the architecture of ECM is one of the key challenges of tissue engineering. Among all the methods used to generate artificial ECM, nanofibrous scaffolds demonstrated the most promising results. Nanofibrous scaffolds, irrespective of their method of synthesis, are characterized by high surface area and enhanced porosity, which are highly desired for tissue engineering and drug delivery applications.

At present, there are three dominant approaches to generate nanofibrous scaffolds: molecular self-assembly, phase separation, and electrospinning. Of these approaches, electrospinning is the most widely studied since it is a simple and versatile technique that can produce fibers with a diameter from the micrometer down to nanometer range. Furthermore, it allows one to tailor many aspects of the resulting scaffolds (Casper et al., 2004; Moroni et al., 2006; Liu et al., 2011): (1) the fiber diameter can easily be controlled by varying the solution properties and the processing parameters, (2) the nanofibers can be collected with a rich variety of aligned/random structures using specially designed collectors, (3) they can be stacked and/or folded to form complex structures or architectures, (4) they can be obtained with a hollow or core-sheath structure by changing the configuration of the spinneret, and (5) nanofibers with porous surface structure can be achieved by altering the parameters such as solvent and humidity. The potential of nanofibrous scaffolds for the regeneration of various tissues is now under extensive investigation. In spite of the great achievements behind the design of nanofibrous scaffolds, there is still plenty of room for improvement.

The future research on nanofibrous architecture may be focused on the following aspects: (1) design the structure of nanofibrous architecture. 
One strategy is to exploit new nanofabrication techniques. Recently, Badrossamay et al. (2010) developed a new technique for generation of continuous fibers by rotary jet-spinning. Using this technique, nanofiber structure can be fabricated into an aligned 3D structure or any arbitrary shape by varying the collector geometry. Another strategy is to integrate nanofiber into microfabricated 3D scaffolds to achieve more desirable scaffolds. For example, co-electrospun nanofluidic channels were integrated into stimuli-sensitive hydrogels to realize controlled release of biomolecules (Yang et al., 2012). A third strategy is to fully explore current approaches of fabricating nanofibrous scaffolds. For instance, highly porous core-shell fiber networks were fabricated using an electrospinning system containing a water-immersed collector (Muhammad et al., 2011). Finally, in combination with nanofabrication technologies, nanofibrous scaffold could be decorated with nanotopographic patterns, such as ridges and grooves, to better match the nanostructure of ECM. (2) Achieve a better control of ECM-mimicry. Nanofibrous scaffolds should be designed more and more as bioactive systems rather than just passive cell carriers. Integration of fabrication techniques with surface modification methods and the achievement of precise positioning of different bioactive cues will be a route to obtain nanofibrous scaffolds with closer properties to native ECM, and (3) Gain a better fundamental understanding of cell-scaffold interaction in vitro, followed by in vivo assessment. There are some important factors, such as mechanical properties and morphological optimization at the nano-, micro- and macroscale that need to be addressed at 'design stage' in tailoring nanofibrous scaffolds. All of these factors play an important role in governing cellular responses and host integration.

Although much joint effort by scientists from multiple disciplines is still needed for the development of nanofibrous scaffolds in different tissue engineering applications, it can be foreseen that nanofibrous scaffolds could approach 'off-the-shelf' surgically implantable constructs in the near future.

\subsection{References}

Agarwal, S., Greiner, A. and Wendorff, J. H. (2009). Electrospinning of manmade and biopolymer nanofibers - Progress in techniques, materials, and applications. Advanced Functional Materials, 19, 2863-2879.

Badrossamay, M. R., McIlwee, H. A., Goss, J. A. and Parker, K. K. (2010). Nanofiber assembly by rotary jet-spinning. Nano Letters, 10, 2257-2261.

Barry, J. J. A., Howard, D., Shakesheff, K. M., Howdle, S. M. and Alexander, M. R. (2006). Using a core-sheath distribution of surface chemistry through 3D tissue engineering scaffolds to control cell ingress. Advanced Materials, 18, 1406-1410.

Casper, C. L., Stephens, J. S., Tassi, N. G., Chase, D. B. and Rabolt, J. F. (2004). Controlling surface morphology of electrospun polystyrene fibers: Effect of 
humidity and molecular weight in the electrospinning process. Macromolecules, 37, 573-578.

Castner, D. G. and Ratner, B. D. (2002). Biomedical surface science: Foundations to frontiers. Surface Science, 500, 28-60.

Chen, F., Lee, C. and Teoh, S. (2007). Nanofibrous modification on ultra-thin poly (e-caprolactone) membrane via electrospinning. Materials Science and Engineering: C, 27, 325-332.

Chua, K. N., Lim, W. S., Zhang, P., Lu, H., Wen, J., Ramakrishna, S., Leong, K. W. and Mao, H. Q. (2005). Stable immobilization of rat hepatocyte spheroids on galactosylated nanofiber scaffold. Biomaterials, 26, 2537-2547.

Chung, C. and Burdick, J. A. (2008). Engineering cartilage tissue. Advanced Drug Delivery Reviews, 60, 243-262.

Cui, H., Muraoka, T., Cheetham, A. G. and Stupp, S. I. (2009). Self-assembly of giant peptide nanobelts. Nano Letters, 9, 945-951.

Cui, H., Webber, M. J. and Stupp, S. I. (2010a). Self-assembly of peptide amphiphiles: From molecules to nanostructures to biomaterials. Peptide Science, 94, 1-18.

Cui, W., Li, X., Chen, J., Zhou, S. and Weng, J. (2008). In situ growth kinetics of hydroxyapatite on electrospun poly (DL-lactide) fibers with gelatin grafted. Crystal Growth and Design, 8, 4576-4582.

Cui, W., Zhou, Y. and Chang, J. (2010b). Electrospun nanofibrous materials for tissue engineering and drug delivery. Science and Technology of Advanced Materials, 11, 014108.

Deitzel, J., Kosik, W., McKnight, S., Beck Tan, N., Desimone, J. and Crette, S. (2002). Electrospinning of polymer nanofibers with specific surface chemistry. Polymer, 43, 1025-1029.

Denes, F. S. and Manolache, S. (2004). Macromolecular plasma-chemistry: An emerging field of polymer science. Progress in Polymer Science, 29, 815-885.

Deng, X. L., Sui, G., Zhao, M. L., Chen, G. Q. and Yang, X. P. (2007). Poly (L-lactic acid)/hydroxyapatite hybrid nanofibrous scaffolds prepared by electrospinning. Journal of Biomaterials Science, Polymer Edition, 18, 117-130.

Gelain, F., Bottai, D., Vescovi, A. and Zhang, S. (2006). Designer self-assembling peptide nanofiber scaffolds for adult mouse neural stem cell 3-dimensional cultures. PLoS One, 1, e119.

Goldberg, M., Langer, R. and Jia, X. (2007). Nanostructured materials for applications in drug delivery and tissue engineering. Journal of Biomaterials Science. Polymer Edition, 18, 241.

Guimond, S., Schütz, U., Hanselmann, B., Körner, E. and Hegemann, D. (2011). Influence of gas phase and surface reactions on plasma polymerization. Surface and Coatings Technology, 205, S447-S450.

Guo, J., Su, H., Zeng, Y., Liang, Y. X., Wong, W. M., Ellis-Behnke, R. G., So, K. F. and Wu, W. (2007). Reknitting the injured spinal cord by self-assembling peptide nanofiber scaffold. Nanomedicine: Nanotechnology, Biology and Medicine, $\mathbf{3}$, 311-321.

Hartgerink, J. D., Beniash, E. and Stupp, S. I. (2001). Self-assembly and mineralization of peptide-amphiphile nanofibers. Science, 294, 1684-1688.

He, C. L., Huang, Z. M., Han, X. J., Liu, L., Zhang, H. S. and Chen, L. S. (2006). Coaxial electrospun poly (L-lactic acid) ultrafine fibers for sustained drug delivery. Journal of Macromolecular Science Part B - Physics, 45, 515-524. 
Holzwarth, J. M. and Ma, P. X. (2011). 3D nanofibrous scaffolds for tissue engineering. Journal of Materials Chemistry, 21, 10243-10251.

Hu, J., Sun, X., Ma, H., Xie, C., Chen, Y. E. and Ma, P. X. (2010). Porous nanofibrous PLLA scaffolds for vascular tissue engineering. Biomaterials, 31, 7971-7977.

Hung, A. M. and Stupp, S. I. (2007). Simultaneous self-assembly, orientation, and patterning of peptide-amphiphile nanofibers by soft lithography. Nano Letters, 7, 1165-1171.

Jiao, Y. P. and Cui, F. Z. (2007). Surface modification of polyester biomaterials for tissue engineering. Biomedical Materials, 2, R24.

Jun, I., Jeong, S. and Shin, H. (2009). The stimulation of myoblast differentiation by electrically conductive sub-micron fibers. Biomaterials, 30, 2038-2047.

Kim, J., Magno, M. H. R., Waters, H., Doll, B. A., McBride, S., Alvarez, P., Darr, A., Vasanji, A., Kohn, J. and Hollinger, J. O. (2012). Bone regeneration in a rabbit critical-size calvarial model using tyrosine-derived polycarbonate scaffolds. Tissue Engineering, 18, 1132-1139.

Kimakhe, S., Bohic, S., Larrose, C., Reynaud, A., Pilet, P., Giumelli, B., Heymann, D. and Daculsi, G. (1999). Biological activities of sustained polymyxin B release from calcium phosphate biomaterial prepared by dynamic compaction: An in vitro study. Journal of Biomedical Materials Research, 47, 18-27.

Kisiday, J., Jin, M., Kurz, B., Hung, H., Semino, C., Zhang, S. and Grodzinsky, A. (2002). Self-assembling peptide hydrogel fosters chondrocyte extracellular matrix production and cell division: Implications for cartilage tissue repair. Proceedings of the National Academy of Sciences, 99, 9996.

Lee, K., Kim, H., Khil, M., Ra, Y. and Lee, D. (2003). Characterization of nano-structured poly ( $\varepsilon$-caprolactone) nonwoven mats via electrospinning. Polymer, 44, 1287-1294.

Li, C., Vepari, C., Jin, H. J., Kim, H. J. and Kaplan, D. L. (2006). Electrospun silk-BMP-2 scaffolds for bone tissue engineering. Biomaterials, 27, 3115-3124.

Li, D., Wang, Y. and Xia, Y. (2004). Electrospinning nanofibers as uniaxially aligned arrays and layer-by-layer stacked films. Advanced Materials, 16, 361-366.

Li, d. and Xia, Y. (2004). Direct fabrication of composite and ceramic hollow nanofibers by electrospinning. Nano Letters, 4, 933-938.

Li, W. J., Tuli, R., Okafor, C., Derfoul, A., Danielson, K. G., Hall, D. J. and Tuan, R. S. (2005). A three-dimensional nanofibrous scaffold for cartilage tissue engineering using human mesenchymal stem cells. Biomaterials, 26, 599-609.

Liu, W., Thomopoulos, S. and Xia, Y. (2012). Electrospun nanofibers for regenerative medicine. Advanced Healthcare Materials, 1(1), 10-25.

Luong, N. D., Moon, I. S., Lee, D. S., Lee, Y. K. and Nam, J. D. (2008). Surface modification of poly (l-lactide) electrospun fibers with nanocrystal hydroxyapatite for engineered scaffold applications. Materials Science and Engineering: $C, \mathbf{2 8}$, 1242-1249.

Ma, P. X. and Zhang, R. (1999). Synthetic nano-scale fibrous extracellular matrix. Journal of Biomedical Materials Research, 46, 60-72.

Ma, Z., Kotaki, M., Yong, T., He, W. and Ramakrishna, S. (2005). Surface engineering of electrospun polyethylene terephthalate (PET) nanofibers towards development of a new material for blood vessel engineering. Biomaterials, 26, 2527-2536. 
Mao, J., Duan, S., Song, A., Cai, Q., Deng, X. and Yang, X. (2012). Macroporous and nanofibrous poly (Lactide-co-Glycolide) (50/50) scaffolds via phase separation combined with particle-leaching. Materials Science and Engineering: $C, 32$, 1407-1414.

Miller, D. C., Thapa, A., Haberstroh, K. M. and Webster, T. J. (2004). Endothelial and vascular smooth muscle cell function on poly (lactic-co-glycolic acid) with nano-structured surface features. Biomaterials, 25, 53-61.

Misawa, H., Kobayashi, N., Soto-Gutierrez, A., Chen, Y., Yoshida, A., Rivas-Carrillo, J. D., Navarro-Alvarez, N., Tanaka, K., Miki, A., Takei, J., Ueda, T., Tanaka, M., Endo, H., Tanaka, N. and Ozaki, T. (2006). PuraMatrix ${ }^{\mathrm{TM}}$ facilitates bone regeneration in bone defects of calvaria in mice. Cell Transplantation, 15, 903-910.

Mo, X. and Weber, H. J. (2004). Electrospinning P (LLA-CL) nanofiber: A tubular scaffold fabrication with circumferential alignment. Macromolecules. Symposia, 21, 413-416.

Morent, R., de Geyter, N., Desmet, T., Dubruel, P. and Leys, C. (2011). Plasma surface modification of biodegradable polymers: A review. Plasma Processes and Polymers, 8, 171-190.

Moroni, L., Licht, R., de Boer, J., de Wijn, J. R. and van Blitterswijk, C. A. (2006). Fiber diameter and texture of electrospun PEOT/PBT scaffolds influence human mesenchymal stem cell proliferation and morphology, and the release of incorporated compounds. Biomaterials, 27, 4911-4922.

Muhammad, G., Lee, J. M., Kim, J., Lim, D. W., Lee, E. K. and Chung, B. G. (2011). Highly porous core-shell polymeric fiber network. Langmuir, 27, 10993-10999.

Neves,S.C.,Moreira Teixeira,L.S.,Moroni,L., Reis, R.L., van Blitterswijk,C.A.,Alves, N. M., Karperien, M. and Mano, J. F. (2011). Chitosan/Poly( $\varepsilon$-caprolactone) blend scaffolds for cartilage repair. Biomaterials, 32, 1068-1079.

Nisbet, D., Forsythe, J., Shen, W., Finkelstein, D. and Horne, M. (2009). Review paper: A review of the cellular response on electrospun nanofibers for tissue engineering. Journal of Biomaterials Applications, 24, 7-29.

Park, H., Cannizzaro, C., Vunjak-Novakovic, G., Langer, R., Vacanti, C. A. and Farokhzad, O. C. (2007a). Nanofabrication and microfabrication of functional materials for tissue engineering. Tissue Engineering, 13, 1867-1877.

Park, K., Ju, Y. M., Son, J. S., Ahn, K. D. and Han, D. K. (2007b). Surface modification of biodegradable electrospun nanofiber scaffolds and their interaction with fibroblasts. Journal of Biomaterials Science, Polymer Edition, 18, 369-382.

Pettikiriarachchi, J. T. S. , Parish, C. L., Nisbet, D. R. and Forsythe, J. S. (2012). Architectural and surface modification of nanofibrous scaffolds for tissue engineering, Available from: http://onlinelibrary.wiley.com/doi/10.1002/ 9783527610419.ntls0258/full (Accessed 19 April 2013).

Philp, D. and Stoddart, J. F. (1996). Self-assembly in natural and unnatural systems. Angewandte Chemie International Edition in English, 35, 1154-1196.

Prabaharan, M., Jayakumar, R. and Nair, S. (2011). Electrospun nanofibrous scaffolds-current status and prospects in drug delivery. Advances in Polymer Science, 246, 241-262.

Qi, Z. D., Saito, T., Fan, Y. and Isogai, A. (2012). Multifunctional coating films by layer-by-layer deposition of cellulose and chitin nanofibrils. Biomacromolecules, 13, 553-558.

Ramakrishna,S.(2005).An Introduction to Electrospinning and Nanofibers, Singapore, World Scientific Pub Co Inc. 
Rhoades, R. and Pflanzer, R. G. (1996). Human Physiology, USA Saunders College Pub.

Sargeant, T. D., Guler, M. O., Oppenheimer, S. M., Mata, A., Satcher, R. L., Dunand, D. C. and Stupp, S. I. (2008). Hybrid bone implants: Self-assembly of peptide amphiphile nanofibers within porous titanium. Biomaterials, 29, 161-171.

Semino, C. E., Kasahara, J., Hayashi, Y. and Zhang, S. (2004). Entrapment of migrating hippocampal neural cells in three-dimensional peptide nanofiber scaffold. Tissue Engineering, 10, 643-655.

Shah, R. N., Shah, N. A., Lim, M. M. D. R., Hsieh, C., Nuber, G. and Stupp, S. I. (2010). Supramolecular design of self-assembling nanofibers for cartilage regeneration. Proceedings of the National Academy of Sciences, 107, 3293-3298.

Shin, M., Yoshimoto, H. and Vacanti, J. P. (2004). In vivo bone tissue engineering using mesenchymal stem cells on a novel electrospun nanofibrous scaffold. Tissue Engineering, 10, 33-41.

Sill, T. J. and von Recum, H. A. (2008). Electrospinning: Applications in drug delivery and tissue engineering. Biomaterials, 29, 1989-(2006.

Silva, G. A., Czeisler, C., Niece, K. L., Beniash, E., Harrington, D. A., Kessler, J. A. and Stupp, S. I. (2004). Selective differentiation of neural progenitor cells by high-epitope density nanofibers. Science, 303, 1352-1355.

Smith, I., Liu, X., Smith, L. and Ma, P. (2009). Nanostructured polymer scaffolds for tissue engineering and regenerative medicine. Wiley Interdisciplinary Reviews: Nanomedicine and Nanobiotechnology, 1, 226-236.

Smith, L. A., Liu, X. and Ma, P. X. (2008). Tissue engineering with nano-fibrous scaffolds. Soft Matter, 4, 2144-2149.

Stitzel, J., Liu, J., Lee, S. J., Komura, M., Berry, J., Soker, S., Lim, G., van Dyke, M., Czerw, R. and Yoo, J. J. (2006). Controlled fabrication of a biological vascular substitute. Biomaterials, 27, 1088-1094.

Subbiah, T., Bhat, G., Tock, R., Parameswaran, S. and Ramkumar, S. (2005). Electrospinning of nanofibers. Journal of Applied Polymer Science, 96, 557-569.

Sui, G., Yang, X., Mei, F., Hu, X., Chen, G., Deng, X. and Ryu, S. (2007). Poly-L-lactic acid/hydroxyapatite hybrid membrane for bone tissue regeneration. Journal of Biomedical Materials Research Part A, 82, 445-454.

Sur, S., Pashuck, E. T., Guler, M. O., Ito, M., Stupp, S. I. and Launey, T. (2011). A hybrid nanofiber matrix to control the survival and maturation of brain neurons. Biomaterials, 33, 545-555.

Tillman, B. W., Yazdani, S. K., Lee, S. J., Geary, R. L., Atala, A. and Yoo, J. J. (2009). The in vivo stability of electrospun polycaprolactone-collagen scaffolds in vascular reconstruction. Biomaterials, 30, 583-588.

Venugopal, J., Low, S., Choon, A. T. and Ramakrishna, S. (2008). Interaction of cells and nanofiber scaffolds in tissue engineering. Journal of Biomedical Materials Research Part B: Applied Biomaterials, 84, 34-48.

Venugopal, J. and Ramakrishna, S. (2005). Applications of polymer nanofibers in biomedicine and biotechnology. Applied Biochemistry and Biotechnology, 125, 147-157.

Wang, M., Jing, N., Su, C. B., Kameoka, J., Chou, C. K., Hung, M. C. and Chang, K. A. (2006). Electrospinning of silica nanochannels for single molecule detection. Applied Physics Letters, 88, 033106. 
Wang, Y., Kim, U. J., Blasioli, D. J., Kim, H. J. and Kaplan, D. L. (2005). In vitro cartilage tissue engineering with $3 \mathrm{D}$ porous aqueous-derived silk scaffolds and mesenchymal stem cells. Biomaterials, 26, 7082-7094.

Watari, S., Hayashi, K., Wood, J. A., Russell, P., Nealey, P. F., Murphy, C. J. and Genetos, D. C. (2011). Modulation of osteogenic differentiation in hMSCs cells by submicron topographically-patterned ridges and grooves. Biomaterials, 33, 128-136.

Woo, K. M., Chen, V. J. and Ma, P. X. (2003). Nano-fibrous scaffolding architecture selectively enhances protein adsorption contributing to cell attachment. Journal of Biomedical Materials Research Part A, 67, 531-537.

Yang, F., Murugan, R., Wang, S. and Ramakrishna, S. (2005). Electrospinning of nano/ micro scale poly (L-lactic acid) aligned fibers and their potential in neural tissue engineering. Biomaterials, 26, 2603-2610.

Yang, H., Hong, W. and Dong, L. (2012). A controlled biochemical release device with embedded nanofluidic channels. Applied Physics Letters, 100, 153510-153510-4.

Yoo, H. S., Kim, T. G. and Park, T. G. (2009). Surface-functionalized electrospun nanofibers for tissue engineering and drug delivery. Advanced Drug Delivery Reviews, 61, 1033-1042.

Yoshimoto, H., Shin, Y., Terai, H. and Vacanti, J. (2003). A biodegradable nanofiber scaffold by electrospinning and its potential for bone tissue engineering. Biomaterials, 24, 2077-2082.

Zhang, Y., Venugopal, J., Huang, Z. M., Lim, C. and Ramakrishna, S. (2005). Characterization of the surface biocompatibility of the electrospun PCL-collagen nanofibers using fibroblasts. Biomacromolecules, 6, 2583-2589.

Zhang, Y., Wang, X., Feng, Y., Li, J., Lim, C. and Ramakrishna, S. (2006). Coaxial electrospinning of (fluorescein isothiocyanate-conjugated bovine serum albumin)-encapsulated poly ( $\varepsilon$-caprolactone) nanofibers for sustained release. Biomacromolecules, 7, 1049-1057.

Zhang, Z., Hu, J. and Ma, P. X. (2012). Nanofiber-based delivery of bioactive agents and stem cells to bone sites. Advanced Drug Delivery Reviews, 64, 1129-1141.

Zhao, Y., Tanaka, M., Kinoshita, T., Higuchi, M. and Tan, T. (2010). Nanofibrous scaffold from self-assembly of [beta]-sheet peptides containing phenylalanine for controlled release. Journal of Controlled Release, 142, 354-360.

Zhong, C., Cooper, A., Kapetanovic, A., Fang, Z., Zhang, M. and Rolandi, M. (2010). A facile bottom-up route to self-assembled biogenic chitin nanofibers. Soft Matter, 6, 5298-5301.

Zhu, X., Chian, K. S., Chan-Park, M. B. E. and Lee, S. T. (2005). Effect of argonplasma treatment on proliferation of human-skin-derived fibroblast on chitosan membrane in vitro. Journal of Biomedical Materials Research Part A, 73, 264-274.

Zhu, Y., Gao, C., Liu, X. and Shen, J. (2002). Surface modification of polycaprolactone membrane via aminolysis and biomacromolecule immobilization for promoting cytocompatibility of human endothelial cells. Biomacromolecules, $\mathbf{3}$, 1312-1319. 

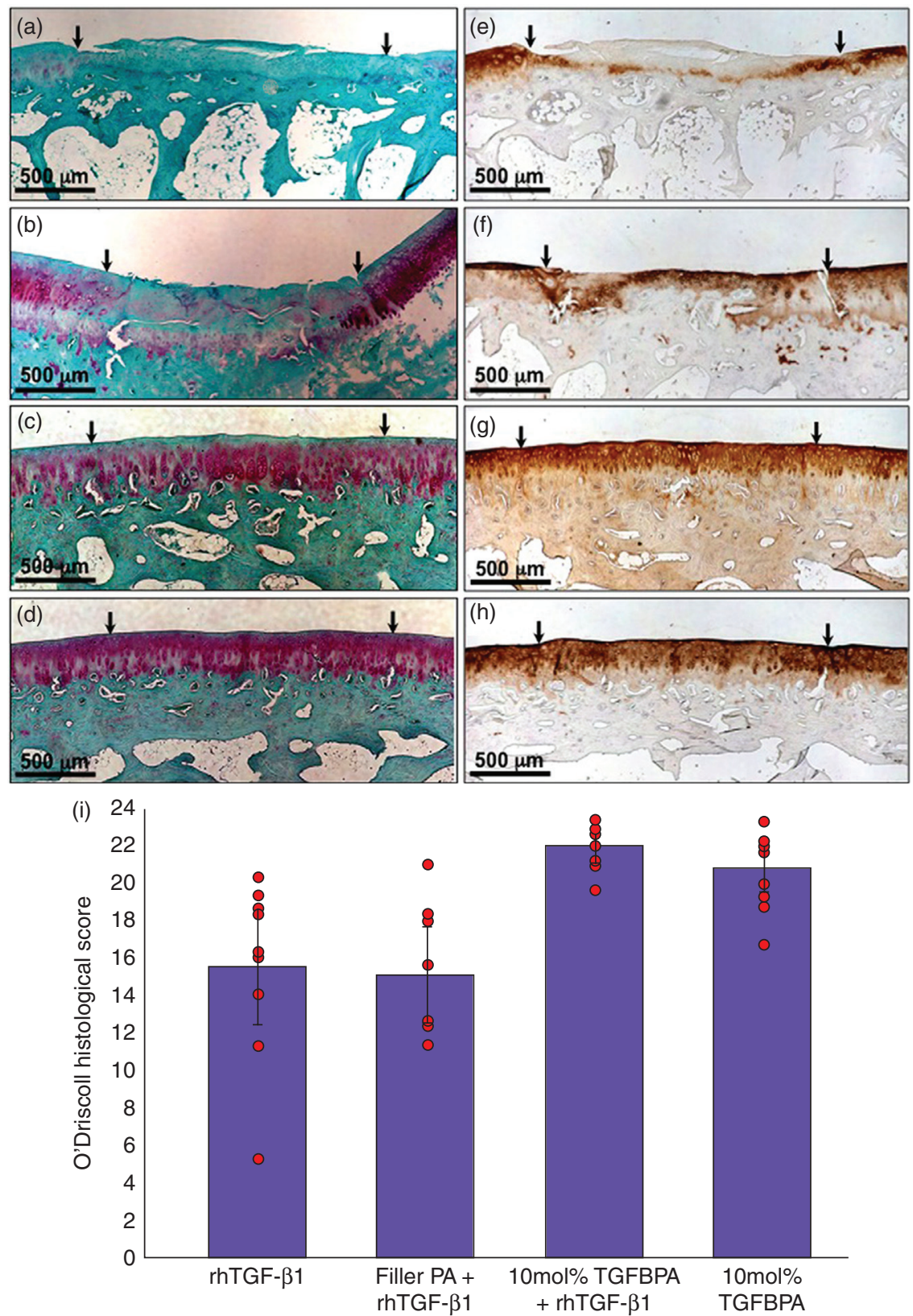

Plate VII (Chapter 6) (a-h) Histological evaluation of sample sections 12 weeks after treatment. Safranin-O staining for glycosaminoglycans (a-d) and type II collagen staining (e-h) in articular cartilage defects treated with $(\mathrm{a}, \mathrm{e}) 100 \mathrm{ng} / \mathrm{mLTGF}-\beta 1$ (100TGF), (b, f) filler PA+TGF- $\beta 1,(\mathrm{c}, \mathrm{g})$ $10 \%$ TGFBPA+TGF- $\beta 1$, and (d, h) 10\%TGFBPA alone 12 weeks post-op; (i) Histological scores of 12 week in vivo samples. Circles represent scores for individual specimens in each group $(n=8-10)$. The arrows show the boundaries of articular cartilage defects before treatment (Shah et al., 2010). 